\title{
LA ELECTRICIDAD EN LAS RELACIONES ESPAÑA-PORTUGAL
}

\author{
Cayetano Espejo Marín ${ }^{1}$
}

\begin{abstract}
Resumen - Desde comienzos de los años 60 del siglo pasado España y Portugal mantienen intensos intercambios de energía eléctrica. Esto ha permitido a los dos países un incremento en el nivel de seguridad en el abastecimiento de electricidad, y la obtención de un mejor aprovechamiento de sus recursos energéticos. Con la puesta en marcha del Mercado Ibérico de Electricidad, prevista para Abril de 2004, se inicia una nueva etapa en las relaciones de España y Portugal. Este nuevo Mercado se construye sobre tres pilares fundamentales: el incremento de las interconexiones eléctricas fronterizas, la creación de un Operador del Mercado Ibérico único, y la coordinación de los dos operadores del sistema actuales, Red Eléctrica de España y Rede Eléctrica Nacional. El Mercado Ibérico de Electricidad será el primero integrado sólo por países miembros de la Unión Europea, cuarto en términos de producción, y representará una décima parte la electricidad consumida en toda Europa.
\end{abstract}

Palabras clave : Electricidad, España, Portugal, compañía eléctrica, Mercado Ibérico de Electricidad.

Resumo - A electricidade nas Relações luso-espanholas. Desde início dos anos 60 do século passado, Espanha e Portugal mantêm intensos intercâmbios de energia eléctrica. Isto permitiu aos dois países um incremento ao nível da segurança no abastecimento de energia eléctrica, e a obtenção de um melhor aproveitamento dos seus recursos energéticos. Com o arranque do Mercado Ibérico de Electricidade, previsto para Abril de 2004, inicia-se uma nova etapa nas relações entre Espanha e Portugal. Este novo Mercado é construído sobre três pilares fundamentais: o incremento das interconexões eléctricas fronteiriças, a criação de um Operador de Mercado Ibérico único, e a coordenação dos dois actuais operadores do sistema, Red Eléctrica de España e Rede Eléctrica Nacional. O Mercado Ibérico de Electricidade será o primeiro a reunir unicamente países da União Europeia, o quarto em termos de produção da UE e representará uma décima parte da electricidade consumida em toda a Europa.

Palavras-chave : Electricidade, Espanha, Portugal, companhia de electricidade, Mercado Ibérico de Electricidade.

1 Profesor Titular de Geografía Humana. Departamento de Geografía, Universidad de Murcia, Campus La Merced, 30001 Murcia, España. E-mail: cespejo@um.es. Manuscrito recebido em Fevereiro de 2004 


\begin{abstract}
Electricity and the Relations between Spain and Portugal. From the early 1960s onwards, Spain and Portugal have maintained far-reaching programs for the exchange of electric power. These contacts have afforded, on the one hand, an increase in the security of electricity supply for both countries and, on the other, a better exploitation of their particular energy resources. The likely launching of the Iberian Electricity Market in April 2004 will lead to a new phase in the relations between Portugal and Spain. The new Market is based on three essential elements: i) the expansion of electric links along their shared national borders, ii) the setting up of a lone Operator for the single Iberian Market and iii) the coordination of the two Operators that exist nowadays, Red Eléctrica de España and Rede Eléctrica Nacional. The Iberian Electricity Market will be the first to comprise countries of the European Community only and it will generate one-tenth of the electric power consumed in Europe, being the fourth largest producer.
\end{abstract}

Key words : Electricity, Spain, Portugal, electricity supply company, Iberian Electricity Market.

Résumé - L'électricité dans les ReLations luso-espagnoles. Depuis le début des années $60 \mathrm{du} \mathrm{XX}^{\mathrm{e}}$ siècle, l'Espagne et le Portugal procèdent à d'importants échanges d'énergie électrique, ce qui a rendu plus sûr leur approvisionnement en électricité et assuré un meilleur équilibre de leurs ressources énergétiqus. La mise en route du Marché Commun Ibérique de l'Électricité, en avril 2004, représente une nouvelle étape dans les relations luso-espagnoles. Ce Marché Commun repose sur trois bases : le développement des interconnexions électriques frontalières, la création d'un Opérateur unique et la coordination des opérateurs des deux systèmes actuels, Red Eléctrica De España e Rede Eléctrica Nacional. Le Marché Ibérique de l'Électricité sera le premier à réunir seulement des pays membres de l'Union Européenne et le quatrième quant à la production, celle-ci représentant le dixième de toute l'électricité consommée en Europe.

Mots-clés: Électricité, Espagne, Portugal, Compagnie électriques, Marché Ibérique de l'Électricité.

\title{
I. INTRODUCCIÓN
}

La puesta en marcha del Mercado Ibérico de la Electricidad (MiBeL), prevista para el mes de Abril de 2004, supone la consolidación y ampliación de las relaciones entre España y Portugal en materia de energía eléctrica, iniciadas a mediados de los años 50 del siglo pasado.

Desde finales de los años 90 se ha producido la adquisición de capital social de algunas empresas eléctricas por parte de otras del país vecino. Es el caso de Iberdrola que posee el 5\% de Electricidade de Portugal, a su vez, esta sociedad cuenta con el 40\% de Hidroeléctrica del Cantábrico, y Endesa, la mayor eléctrica española, también tiene presencia en Portugal, con un 44,9\% de Pego Energía Eléctrica.

En este artículo se analiza la evolución de los intercambios de electricidad entre los dos países ibéricos desde que se inician, la participación de las empresas eléctricas en las del país vecino, y en tercer lugar los objetivos del MibeL. 


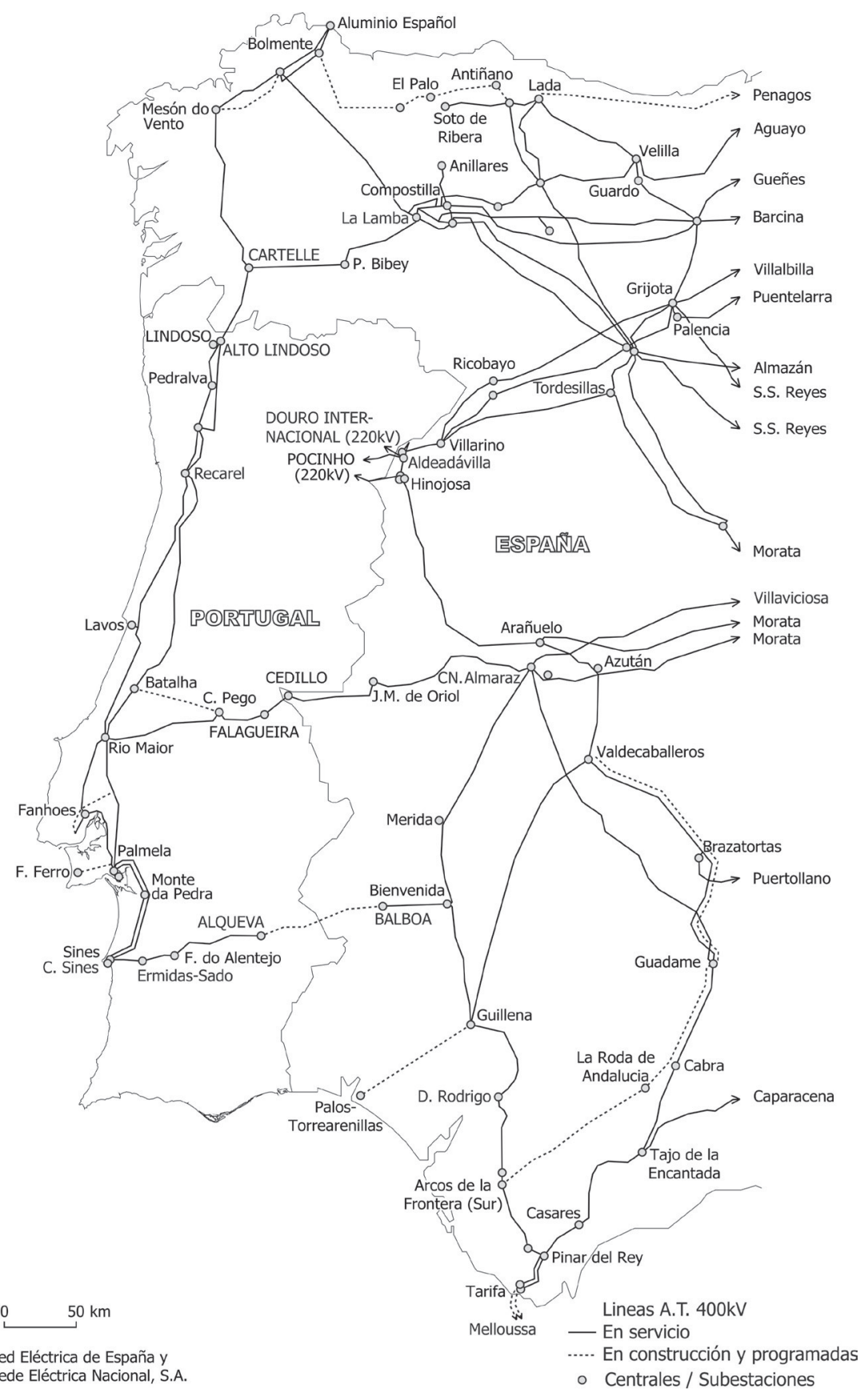

Fig. 1 - Red española peninsular de transporte de electricidad.

Fig. 1 - Rede espanhola peninsular de transporte de electricidade.

Fig. 1 - Iberian Peninsular electricity network. 


\section{INTERCAMBIOS DE ELECTRICIDAD ENTRE ESPAÑA Y PORTUGAL}

La red española peninsular de transporte de electricidad se encuentra directamente interconectada con las de Francia, Portugal, Andorra y Marruecos (fig. 1), de forma que España viene realizando intercambios internacionales desde mediados de los años cuarenta del siglo pasado. El objetivo de estos intercambios ha sido el incremento del nivel de seguridad en el abastecimiento de electricidad y la obtención de un mejor aprovechamiento de los recursos existentes. Permiten que los países interconectados se apoyen coyunturalmente cuando surgen problemas de suministro, derivados, por ejemplo, de averías que reducen la disponibilidad de determinadas centrales en un momento dado. Por otro lado, la posibilidad de contar con recursos hídricos, las variaciones de la demanda, y las diferencias en el coste de producción del kilovatio/hora (kWh), justifican también estos intercambios de energía eléctrica, que a su vez posibilitan una mejora de la gestión de los sistemas eléctricos de cada país.

\section{$10^{9} \mathrm{kWh}$}

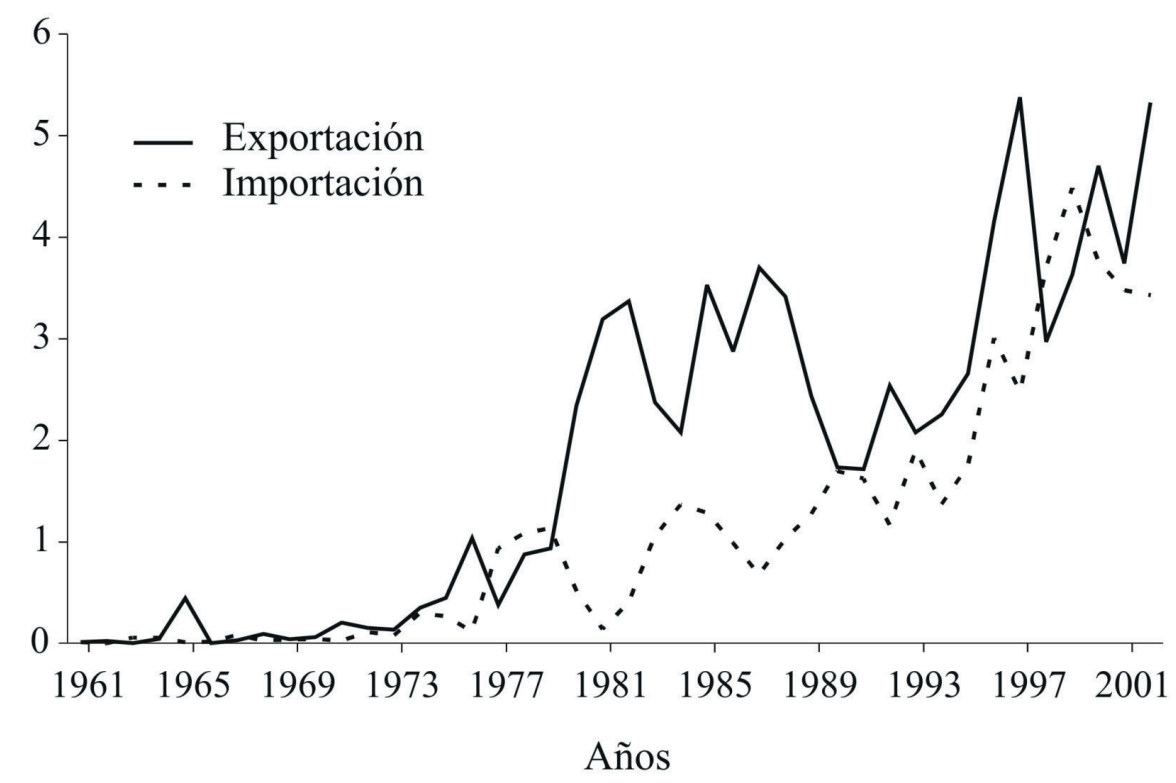

Fuente: Memoria Estadística. Informe sobre las Actividades Eléctricas. UNESA, 2002.

Fig. 2 - Intercambio de energía eléctrica España-Portugal, 1961-2002.

Fig. 2 - Intercâmbio de energia eléctrica entre Espanha e Portugal, 1961-2002.

Fig. 2 - Interchange of electrical energy Spain-Portugal, 1961-2002. 
En la actualidad, las interconexiones internacionales representan un elemento importante para la seguridad del suministro eléctrico. Además, mejoran la estabilidad de la red y el mantenimiento de la frecuencia y de la tensión. También son un elemento esencial para que los intercambios de energía permitan una mayor competencia en los sistemas interconectados.

Aunque durante los años 1953 y 1955 España realiza ventas de electricidad a Portugal, no es hasta 1961 cuando de un modo regular se produce el intercambio de este tipo de energía (fig. 2). A finales de los años 50 España pone en marcha el Plan de Estabilización y poco después inicia su primer Plan de Desarrollo. Por otro lado, Portugal inicia antes esa política de desarrollo pues a través del I Plan de Fomento (1953-1958), que preveía una inversión de casi 8 millones de contos, se hicieron prioridades de fomento agrícola, de producción de energía eléctrica, de prolongación de vías de comunicación y de medios de transporte (ANTÚNEZ, 1997).

España y Portugal comparten dos de los ríos más caudalosos de la Península, Duero y Tajo. El Duero se encaja en un abrupto congosto, los Arribes, por donde abandona la Meseta perdiendo bruscamente altura hacia las bajas tierras portuguesas. El aprovechamiento de este tramo fronterizo dio motivo a un acuerdo entre los dos países ibéricos. Como señala R. F. Moreira da Silva, Ainda em relação ao Douro Internacional e no domínio dos aproveitamentos hidroeléctricos, em 16 de Julho de 1964, em Lisboa, foi aprovado um novo Convénio Luso-Espanhol em que é regulado o aproveitamento hidroeléctrico dos Troços Internacionais do Douro e seus afluentes. Só em 1968 um novo Convénio vem regular o uso e aproveitamento hidroeléctrico dos Troços Internacionais dos rios Lima, Minho, Tejo, Guadiana, Chança e seus afluentes. Como nos refere o documento oficial a partilha tem finalidades prioritariamente hidroeléctricas. Cada país adoptou o esquema que considerou mais adequado às regras. Assim, a Portugal foi atribuída a totalidade dos troços internacionais do Guadiana e Lima e a Espanha os de Tejo e Chança (Moreira Da Silva, 1996).

Los intercambios de electricidad de España y Portugal han sido posibles gracias al enorme crecimiento de la potencia instalada a partir de mediados de los años 50. Entre 1957 y 1973, C. SudRIÀ (1990) establece dos etapas en el desarrollo de la energía eléctrica en España. La primera comprende desde 1957 hasta 1964, con un aumento de la potencia instalada total de un 8,5\% anual. En esta etapa se produce la última gran expansión hidroeléctrica. La potencia instalada en este tipo de centrales durante estos años asciende a 3.000 megavatios (MW), casi doblando la capacidad productiva anterior. Para abastecer la creciente demanda de electricidad se incrementa la potencia de generación térmica, tanto en centrales de carbón, como de combustibles derivados del petróleo. La segunda etapa comprende de 1964 a 1973, año de la crisis del petróleo y las consecuencias derivadas de la misma. Durante estos años se produce un gran crecimiento de la capacidad de producción eléctrica, centrada preferentemente en plantas térmicas, cuya potencia se multiplica por tres en estos años. Además empieza a tener importancia la aportación de las primeras centrales nucleares (ESPEJo, 2002). 
Las relaciones del Sistema Eléctrico Español con los sistemas europeos alcanzan su madurez en 1985 de la mano del nuevo concepto de unificación de explotación, introducido con la Ley 49/1984 sobre Explotación Unificada del Sistema Eléctrico Nacional, que le suministra una capacidad de decisión semejante a la de sus vecinos franceses y portugueses. En 1986 se establece un acuerdo marco entre Electricité de France, Red Eléctrica de España y Electricidade de Portugal para el suministro de energía a esta última por parte de Electricité de France y Red Eléctrica Española que, de forma solidaria, garantizan a la eléctrica portuguesa una potencia de $200 \mathrm{MW}$, ampliable a $400 \mathrm{MW}$, que puede ser suministrada desde el sistema más competitivo en cada momento. Este acuerdo dura hasta 1989, cuando la bajada de precios del fuel-oil en el mercado internacional hace que la diferencia de precios de producción casi desaparezca y con ello el interés económico de los intercambios previstos en este acuerdo marco (FABRA, 2004).

En Enero de 1990 las tres sociedades eléctricas firman un acuerdo de intercambios de energía en el cual Red Eléctrica de España constituye el eje de la programación de las tres empresas. Es el Contrato Tripartito Francia-EspañaPortugal el que se adelanta en varios meses a la primera directiva comunitaria relativa al mercado eléctrico europeo, la Directiva de Tránsito. Mediante este acuerdo, que da comienzo el 1 de Julio de 1994, y que tendrá una duración de cinco años, Electricité de France pone a disposición de Red Eléctrica Española una potencia de $385 \mathrm{MW}$, quien a su vez facilita a Electricidade de Portugal 301MW. En ambos casos se permite una interrumpibilidad de un máximo de 200 horas al año. Así, Francia y España satisfacen los requerimientos de Portugal con todo respeto para las leyes físicas que gobiernan la electricidad y con márgenes de beneficio equitativamente distribuidos. El contrato tripartito reconoció el papel que cada compañía eléctrica y cada sistema cumplían en la satisfacción de las necesidades portuguesas y por ello una negociación entre Electricité de France y Electricidade de Portugal que tenía como objeto el establecimiento de un contrato bilateral al que Red Eléctrica de España prestaría un servicio de tránsito, se convirtió en un contrato en el la empresa española suministraba a la portuguesa la energía previamente tomada de la francesa en la frontera pirenaica. La integración de los sistemas del sudoeste europeo y, en particular, la integración de los sistemas de la Península Ibérica dio un fuerte paso hacia delante (FABRA, 2004).

Los intercambios de electricidad entre España y Portugal se realizan a través de dos líneas de $400 \mathrm{~kW}$ y tres líneas de $220 \mathrm{~kW}$, a las que corresponde una capacidad térmica máxima de unos 3.300MW (fig. 1).

La planificación energética española para la primera década del siglo XXI queda recogida en el documento Planificación de los Sectores de Electricidad y Gas. Desarrollo de las Redes de Transporte 2002-2011, aprobado por el Gobierno Español en Septiembre de 2002. En el mismo se contemplan las actuaciones previstas para el desarrollo de las interconexiones entre el sistema eléctrico español y el sistema eléctrico portugués. Los estudios realizados por Red Eléctrica de 
España y Rede Eléctrica Nacional, sobre el desarrollo de la interconexión tienen como conclusión más relevante el nuevo eje previsto Balboa-Alqueva de 400kW. A continuación se relacionan el conjunto de actuaciones previstas para el desarrollo de la interconexión España-Portugal, recopiladas en el Protocolo para la Creación del Mercado Ibérico, de noviembre de 2001:

- Alqueva-Balboa de 400kW, al sur de Extremadura. La nueva línea ofrece unos beneficios económicos de operación asociados a minimización de pérdidas de transporte y contribución al aumento de la capacidad de interconexión.

- Incremento de capacidad de líneas existentes (eje Falagueira-CedilloOriol-Arañuelo/Almaraz 400kW), al norte de Extremadura.

— Eje de 400kW Cartelle-Lindoso, en Galicia (segundo circuito de la línea actual). Esta conexión a $400 \mathrm{~kW}$ por el norte está preparada para ampliación a través del tendido de un segundo circuito.

- Eje 400kW Duero. Existe una previsión para reforzar este corredor con una conexión de $400 \mathrm{~kW}$; esta medida, que para el sistema español es muy factible (conexión muy corta desde la subestación de Aldeadávila) ha encontrado dificultades (medioambientales en el tramo portugués) cuya superación está pendiente de evaluación. Previa a la consecución del corredor en $400 \mathrm{~kW}$, especialmente por la mayor dificultad de 'acercar' a la frontera este nivel de tensión desde el sistema portugués, se prevé una fase con funcionamiento a $220 \mathrm{~kW}$ de la infraestructura diseñada para 400kW (doble circuito Duero Internacional-Aldeadávila).

\section{ADQUISICIÓN DE CAPITAL DE LAS COMPAÑÍAS ELÉCTRICAS IBÉRICAS}

En el año 1998 Iberdrola, la segunda eléctrica española, entra en el capital de la petrolera portuguesa Galp Energía y adquiere el 5\% de Electricidade de Portugal.

En Febrero de 2002 el estado portugués accede a ofrecer a Iberdrola una participación relevante en dos distribuidoras de gas (Beiragas y Tagusgas), ubicadas en la zona fronteriza con Extremadura y Castilla y León, más una cantidad no desvelada, a cambio del $4 \%$ que la eléctrica española posee en Galp. En la primera sociedad Iberdrola tendrá el 58,8\% y el 40,9\% en la segunda. Las distribuidoras ocupan una región del país que representa el 30\% del territorio y el $16 \%$ de la población total. Entre las dos suman cerca de 16.000 clientes (MONForte, 2004).

Este acuerdo responde al interés de Iberdrola por estar presente en el mercado portugués con vistas a la puesta en marcha del Mibel. Iberdrola vendió en Portugal durante 2003 un total de 492GWh a 132 puntos de suministro pertenecientes a clientes industriales. Coincidiendo con el anuncio del acuerdo 
Iberdrola hizo pública la adjudicación a las empresas portuguesas Solicabel, Cabelte, Cunhabarros, Efacec, Siemens Portugal y Actaris Portugal, de un total de diez contratos para el suministro de equipos y materiales destinados al área de distribución y generación, por un importe aproximado de 14 millones de euros (IBERDROLA, 2004).

El 40\% del capital de Hidroeléctrica del Cantábrico es propiedad de Electricidade de Portugal. Esta posición va a permitir a esta empresa ganar en España al menos la cuota de mercado que pudiera perder en su país, con la entrada en funcionamiento del Mibel. Ambas compañías eléctricas suman una cuota de generación del $16 \%$ en el mercado ibérico y del $22 \%$ en comercialización.

Hidrocantábrico tiene en proyecto tres nuevas inversiones para ampliar su capacidad productiva: dos centrales de ciclo combinado y una grupo térmico. Para Electricidade de Portugal, Hidrocantábrico no sólo constituye un elemento de oportunidad, que le ha permitido hacerse con el 5\% del mercado español, sino también, por sus respectivas estructuras productivas, un buen complemento para equilibrar su estructura de generación. La eléctrica portuguesa es muy dependiente de la producción hidráulica, mientras que Hidrocantábrico tiene un fuerte componente térmico, además de su presencia en el sector nuclear, ya que posee el $15 \%$ de la central de Trillo, la titularidad de activos hidráulicos, y una central de ciclo combinado en Castejón (Navarra), inaugurada en 2002.

Para Electricidade de Portugal la idoneidad de su alianza con Hidrocantábrico también se extiende al gas. Con la integración en la eléctrica lusa de los activos gasistas, antes en manos de la petrolera pública Galp, esta reordenación ha supuesto que ambas empresas eléctricas alcancen conjuntamente una posición destacada en el ámbito gasístico ibérico, y cuya liberalización está aún pendiente. En 2003 Hidrocantábrico ha adquirido la mayoría del capital de la operadora gasísta vasca Naturcorp. Para la agrupación de todas las actividades de distribución y transporte se ha constituido una nueva sociedad, denominada Naturcorp I, fruto de la fusión de las sociedades filiales: Naturcorp I, Naturcorp Servicios, Gas de Asturias, Sociedad de Gas de Euskadi, Gas de Figueres y Donostigas. El nuevo grupo resultante se configura como el tercer operador principal en el sector del gas natural, detrás del grupo Gas Natural-Repsol-YPF, y Enagás.

Desde la entrada de Electricidade de Portugal en el capital Hidrocantábrico, durante casi dos años el Gobierno español ha bloqueado sus derechos políticos en la empresa participada, alegando como razones la presencia pública en el capital de la eléctrica portuguesa y la escasa liberalización del sector eléctrico portugués. La restricción queda levantada a finales de Septiembre de $2003^{2}$, quizás como una concesión en medio del diálogo entre ambos gobiernos para la formación del MiBeL.

Endesa, la mayor compañía eléctrica española, también tiene presencia en Portugal. Posee un 44,9\% de Pego Energía Eléctrica, que opera a través de cen-

2 Boletín Oficial del Estado, n. ${ }^{\circ}$ 253, 22 de Octubre de 2003, pp. 37.861-37.863. 
trales termoeléctricas, un 35\% de Tejo Energía, productora y distribuidora, y un $18 \%$ de Carbopego, dedicada al transporte y almacenamiento de combustible.

Además, Endesa tiene una alianza con el grupo local Sonae para constituir la sociedad Sodesa, dedicada a la comercialización de energía.

Durante 2002 exportó $881 \mathrm{GWh}$, lo que supone un $20 \%$ del total de las exportaciones de electricidad realizadas desde España. Estas operaciones tuvieron como destino el suministro a 547 clientes de Endesa Energía en el extranjero, de los que 275 son de Portugal, y operaciones de venta al por mayor a otros sistemas.

\section{EL MERCADO IBÉRICO DE LA ELECTRICIDAD}

El 14 de Noviembre de 2001, el vicepresidente segundo del Gobierno Español y Ministro de Economía, y el Ministro de Economía de Portugal firman en Madrid el Protocolo de colaboración entre las Administraciones española y portuguesa para la creación del Mercado Ibérico de Electricidad. Se establece que el día 1 de Enero de 2003 entrará en funcionamiento el MIBEL, garantizando a todos los agentes establecidos en ambos países el acceso al Operador del Mercado Ibérico y a las interconexiones con terceros países, en condiciones de igualdad y libertad de contratación bilateral. En su artículo $5^{\circ}$, el protocolo establece que las autoridades reguladoras de los sectores eléctricos de España y Portugal deberán presentar, antes del 31 de Marzo de 2002, un modelo de organización del MIBEL que tenga en cuenta los objetivos arriba mencionados, la legislación comunitaria aplicable, la experiencia reciente en funcionamiento de los mercados eléctricos de ambos países y las buenas prácticas de regulación, debiendo las autoridades reguladoras contar con la participación de asociaciones de consumidores, productores, distribuidores, comercializadores, operadores de sistema, operadores de mercado y demás partes interesadas en el desarrollo de dicho Mercado.

En referencia a los objetivos y principios, este Protocolo caracteriza al Mibel del siguiente modo:

- Entra en funcionamiento el día 1 de Enero de 2003.

- Permite el acceso a todos los participantes en condiciones de igualdad, transparencia y objetividad.

- Garantiza a todos los agentes establecidos en ambos países el acceso al Operador de Mercado Ibérico y a las interconexiones con terceros países, en condiciones de igualdad y libertad de contratación bilateral.

- Se rige por los principios de la libre competencia, transparencia, objetividad y eficiencia.

- Es fluido y eficaz, está dotado de los mecanismos de seguimiento y control necesarios para garantizar la satisfacción de las necesidades de los consumidores, la seguridad del abastecimiento a largo plazo y la plena compatibilidad con los objetivos de eficiencia energética y fomento de las energías renovables en ambos países. 
El Protocolo establece además que:

- El Operador del Mercado Ibérico actúe de forma rigurosamente imparcial y su capital esté abierto a las empresas de ambos países.

- Se refuercen las interconexiones eléctricas entre España y Portugal.

- Se eliminen potenciales barreras legislativas y administrativas al pleno desarrollo del MiBeL.

- Se realicen las adaptaciones legislativas necesarias para asegurar la homogeneidad de las condiciones de gestión y funcionamiento de los agentes económicos, de modo que se asegure el pleno ejercicio de la libertad empresarial, en el respeto de los principios económicos comunes.

- La Red Eléctrica Española y la Rede Eléctrica Portuguesa armonicen los procedimientos que permitan la explotación conjunta de los dos sistemas en condiciones óptimas de eficiencia, economía y seguridad.

- Las autoridades reguladoras de los dos países acordarán el reparto de los costes asociados a la implementación del Mibel por parte de los operadores de ambos países.

Para dar respuesta a la solicitud presentada por los gobiernos de España y Portugal, La Comisión Nacional de Energía y la Entidade Reguladora dos Serviços Energéticos elaboran en Marzo de 2002 el documento Modelo de Organización del Mercado Ibérico de Electricidad, en cuyos capítulos, se tratan los siguientes temas: principios de organización y regulación del MibeL, breve evaluación de la situación actual del sector eléctrico en España y Portugal, principales opciones que configuran el modelo de organización del MiBeL, organización inicial del Mibel, tarifas, costes de transición a la competencia y contratos de adquisición de energía, monitorización del funcionamiento del mercado, gestión técnica del sistema de transporte, producción en régimen especial, actividades necesarias para la implementación y desarrollo del MiBeL, y por último puntos a considerar por los gobiernos de España y Portugal.

En el documento, del breve análisis del sector eléctrico en España y Portugal se obtienen las siguientes conclusiones:

- Inexistencia de desequilibrios estructurales, tanto a nivel individual como en el conjunto de los dos sistemas. A pesar de la diferencia de dimensiones (casi de 5 a 1), los dos sistemas presentan estructuras y trayectorias análogas, lo que facilita la construcción del MiBEL.

- Crecimiento muy elevado del consumo de energía eléctrica y de punta (potencia máxima), lo que obliga a proceder a importantes inversiones en nueva capacidad de producción.

- Aumento muy elevado de la inversión subvencionada - directa o indirectamente - en nueva capacidad de producción de energía eléctrica a partir de fuentes de energía renovables y de plantas de cogeneración (producción en régimen especial). 
- Necesidad de considerar y armonizar las reglas de recuperación de inversiones de los productores en régimen ordinario, garantizando que la efectiva recuperación de los costes de transición a un entorno de mercado no distorsione la competencia entre productores ni la formación de los precios de la energía eléctrica en el mercado.

- El panorama empresarial se caracteriza por la presencia de cuatro grandes grupos, lo que obliga a prever mecanismos de control y de actuación adecuados, así como incentivos eficaces para el desarrollo de la competencia entre ellos y para la entrada de nuevos operadores. Es, sin embargo, positivo que algunos de estos grupos hayan comenzado su 'iberización' antes incluso de la firma del protocolo mediante la adquisición de activos, la inversión en nuevas centrales eléctricas, el comercio mayorista transfronterizo y la venta a clientes finales en ambos países.

- Necesidad de acelerar la construcción de nuevas líneas de interconexión y de reforzar las existentes. Las actuales capacidades de interconexión son insuficientes para el desarrollo, no solo del MibeL, sino también del mercado interior de la energía.

En cuanto al Operador del Mercado Ibérico (OMI), será la entidad responsable de la gestión de los mercados diario, intradiario y a plazo. Sus funciones incluyen la aceptación de las ofertas de compra y venta de energía de los agentes de mercado, la casación de estas ofertas y la liquidación de las transacciones efectuadas en los mercados que gestiona, con base en los programas de compra y venta de energía. El OMI será único y llevará a cabo sus actividades en régimen de exclusividad. Su actividad estará regulada y se regirá por los principios de transparencia, objetividad e independencia.

Por otro lado, los dos Operadores de Sistema, actualmente existentes en ambos países, Red Eléctrica de España y Rede Eléctrica Nacional, siguen siendo los responsables de la seguridad y gestión técnica del sistema de cada una de sus áreas de control.

En referencia a las estructuras tarifarias de Portugal y de España deben establecerse de acuerdo a los siguientes principios:

- Igualdad de trato y de oportunidades.

- Transparencia y sencillez en la formulación y establecimiento de las tarifas.

- Eficiencia en la asignación de costes, asegurando la inexistencia de subvenciones cruzadas.

- Eficiencia económica en la utilización de las redes y de la energía eléctrica.

- Contribución a la mejora de las condiciones medioambientales, permitiendo, concretamente, una mayor transparencia en la utilización de las energías renovables y endógenas así como la planificación y gestión de los recursos energéticos. 
Además de los productores en régimen ordinario, existen productores en régimen especial, a los que se les atribuye una licencia especial de producción, para la explotación de energías renovables (especialmente, instalaciones minihidráulicas, parques eólicos, centrales de biomasa, centrales solares o de aprovechamiento de la energía de las olas) o instalaciones de cogeneración. La energía generada por estos productores se remunera en Portugal a través de tarifas predefinidas y en España adoptando una metodología basada en un precio medio de la pool aumentado con una prima por tecnología. Dado el elevado grado de penetración de estos productores en el sistema eléctrico ibérico, es importante analizar la adaptación de los actuales sistemas de incentivos de forma que se facilite su inserción efectiva en el mercado, especialmente a través de la creación de un mercado ibérico de certificados verdes.

Por último, conscientes de su responsabilidad para el éxito del MIBEL, la Comisión Nacional de Energía y la Entidade Reguladora dos Serviços Energéticos entienden que es su deber someter a la consideración de los gobiernos de España y de Portugal algunos puntos que no se contemplan explícitamente en el Protocolo de 14 de Noviembre de 2001 y que necesitan una decisión urgente:

a) Constitución del Operador del Mercado Ibérico. El OMI deberá desarrollar las plataformas necesarias para el desarrollo de los mercados organizados (diario, intradiario, a plazo), proceder a su implementación, prueba y divulgación, de forma que sea posible poner en marcha el MIBEL el 1 de Enero de 2003.

b) Organización de la elegibilidad de los consumidores de baja tensión. Es recomendable la ampliación simultánea de la elegibilidad a todos los consumidores en ambos países. Para que el MIBEL funcione plenamente con éxito es importante que el sistema que se vaya a introducir facilite el cambio de suministrador en condiciones idénticas para todos los consumidores españoles y portugueses.

c) Supervisión del MIBEL. La supervisión eficaz del MIBEL requiere una estrecha colaboración entre varias autoridades reguladoras de España y Portugal.

d) Liberalización del mercado de gas natural. Para que la competencia entre productores de energía eléctrica a partir del gas natural, concretamente en instalaciones de ciclo combinado, no esté falseada, es indispensable que las condiciones de acceso a las infraestructuras de transporte del gas natural en España y Portugal sean equivalentes.

e) Armonización de las condiciones de la producción en régimen especial y de los aprovechamientos hidroeléctricos en cascada. Dado que la producción en régimen especial está llamada a desempeñar un papel de creciente importancia en la cobertura de la demanda de energía eléctrica en la Península Ibérica, es importante que los mecanismos competitivos y los incentivos utilizados en España y Portugal sean coherentes y no distorsionen la competencia en el MIBEL. 
f) Fiscalidad. Hay que armonizar la fiscalidad aplicable a las empresas y a los consumidores de energía eléctrica en España y Portugal, eliminando potenciales distorsiones de la competencia en los mercados energéticos.

El 7 de Noviembre de 2003 se celebra en Figueira da Foz (Portugal) la XIX Cumbre Luso-Española en la que ambos países suscriben un Memorando de Comprensión por el que se establecen las condiciones necesarias para la puesta en marcha del MIBEL. De acuerdo con las conclusiones allí alcanzadas, España y Portugal han fijado como fecha de inicio del MIBEL el próximo 20 de Abril de 2004.

En una primera fase, los operadores del mercado de ambos países se mantendrán separados, atribuyéndose la gestión del mercado spot (diario e intradiario) al polo español a través de Compañía Operadora del Mercado Español de Electricidad, S.A. (OMEL), ocupándose el polo portugués, a través del recientemente constituido Operador do Mercado Ibérico de Energía, S.A. (OMIP), del mercado a plazo (a un año máximo). En una fase posterior, tras el análisis de los resultados obtenidos, se determinará la posibilidad de implantación del mercado a plazo puramente financiero.

Con fecha 10 de Diciembre de 2003, el Consejo de Administración de OMEL ${ }^{3}$ aprueba por unanimidad ejecutar el intercambio accionarial entre OMIP $^{4}$ y OMEL, de un $10 \%$ de su capital social, respectivamente. La adquisición del $10 \%$ del capital social de OMIP por parte de OMEL se realiza mediante compraventa de acciones a su actual accionista único, Rede Eléctrica de Portugal.

3 La composición accionarial de OMEL, según el Informe Anual de 2002, la forman cuatro grupos de accionistas. El primer grupo está compuesto por Endesa, Iberdrola, Unión Fenosa e Hidroeléctrica del Cantábrico, con una participación cada sociedad del 5,71\%. El segundo grupo está compuesto por Gas natural y Repsol Petróleo, con el 5,71\% y 2,86\% respectivamente. En el tercer grupo lo integran entidades bancarias BNP Paribas (10\%), SCH (6\%), BBVA (5\%), Ibercaja (5\%), AFI (5\%), SCH Investment (4\%) y BBK (3\%). El cuarto grupo está compuesto por empresas dedicadas a distintas actividades: FC\&M, Sociedad Rectora del mercado de Futuros y Operaciones sobre Cítricos (10\%), Asociación Española de la Industria Eléctrica (5,71\%), Abengoa (5\%), Samca $(2,86 \%)$, Compañía Informática de Bolsa $(2,67 \%)$, Mina Pública Aguas de Terrassa $(2,34 \%)$ y Asociación Española de Fabricantes de Automóviles y Camiones (2\%).

${ }^{4}$ OMIP es una sociedad anónima de nacionalidad portuguesa, constituida el 16 de Junio de 2003, con sede social el Lisboa. Esta sociedad tiene por objetivo social la organización y gestión de un sistema de soporte para la realización de transacciones y liquidaciones en el ámbito del mercado ibérico de energía, cuyas competencias son: la gestión del mercado organizado de la contratación de energía a plazo, la intermediación de los agentes a efectos de relaciones comerciales en el mercado ibérico de electricidad, la gestión de otros mercados de productos de base energética, la prestación de servicios de liquidación en el ámbito de los mercados organizados de energía, la prestación de servicios de liquidación para transacciones estandarizadas en mercados no organizados de energía, y la prestación de servicios de organización de los mercados en el ámbito de operación del sistema eléctrico.

El capital social de OMIP es de un millón de euros, y ha sido desembolsado por la sociedad portuguesa Rede Eléctrica Nacional, concesionaria de la Red de Transporte de Energía Eléctrica en Portugal. 
El 20 de Enero de 2004 los presidentes de gobierno de España y Portugal firman en Lisboa el acuerdo que formaliza el establecimiento del Mibel, que estará vigente a partir del día 20 de Abril de 2004. Las dificultades existentes han provocado el retraso, ya que el protocolo firmado por los dos países en Noviembre de 2001 preveía la puesta en marcha el 1 de Enero de 2003. La unión total no llegará hasta 2006, según los acuerdos entre España y Portugal. En ese año los operadores de mercado de ambos países se fusionarán, después de un proceso de intercambio de participación que se iniciará en 2004 con el canje de un $10 \%$ del capital.

El MIBEL se construye sobre la base de tres pilares fundamentales: el incremento de las interconexiones eléctricas fronterizas, la creación de un Operador del Mercado Ibérico único, y la coordinación de los Operadores del Sistema Red Eléctrica de España y Rede Eléctrica Nacional. En el primer punto, las interconexiones eléctricas aumentarán desde los 700MW del año 2001, hasta los 1.700MW en 2006, con unas inversiones superiores a los 10.000 millones de euros.

Todas las centrales eléctricas de la Península competirán en el nuevo mercado, en el que el operador sólo permitirá producir a las plantas con los precios más bajos. Para que exista igualdad de oportunidades, deben equipararse las indemnizaciones que reciben las empresas españolas por la liberalización (los costes de transición a la competencia) con las que se otorgarán a Electricidade de Portugal, que tiene que romper sus contratos de venta a largo plaza de energía para jugarse las ventas cada día en el mercado (CASADO, 2004).

El MIBEL nace como el primer mercado de electricidad integrado en exclusiva por miembros de la Unión Europea. Después del creado por Suecia, Dinamarca y Noruega, con un número muy inferior de clientes, la Península Ibérica da el primer paso para la constitución de un mercado único al que podrán acceder más de 50 millones de consumidores. Con ello se dan las condiciones para atraer nuevos agentes: productores de energía eléctrica, operadores de mercado, empresas de transporte y distribución de energía, y comercializadores. Además, a partir de ahora, será posible la actuación de agentes en ambos países con igualdad de derechos y obligaciones.

En nuevo mercado está abierto a las seis empresas españolas y tres portuguesas que generan energía eléctrica, aunque cuatro de ellas, Endesa, Iberdrola, Unión Fenosa y Electricidade de Portugal, controlan el 75\% de la producción y el 93\% de la distribución. Estas empresas han comprometido inversiones anuales por 3.700 millones de euros que se sumarán a las inversiones públicas en desarrollo de interconexiones programadas en 10.000 millones de euros, con el fin de que el MIBEL alcance su plena operatividad en Enero de 2006 (Eguibide, 2004).

La creación del MIBEL dará lugar al cuarto mercado europeo en términos de producción, representará el $10 \%$ de la energía consumida en toda Europa y facturará entre 19 y 20 millones de euros anuales. Igualmente, al traer consigo la liberalización de la electricidad en el territorio ibérico, se espera que la competencia se traduzca en una bajada del precio de la luz para particulares y 
empresas, según las autoridades. Esta bajada se prevé que sea más significativa en Portugal, donde ha existido una situación de casi monopolio y donde ahora competirán nueve empresas de generación. Además, con este acuerdo los usuarios podrán cambiar con mayor frecuencia de operador. Para que se de esta situación tendrá que pasar un tiempo, ya que mientras en España los clientes pueden elegir su compañía eléctrica, en Portugal todos tendrán ese derecho en Julio de 2004. Los gobiernos tendrán que establecer unas condiciones similares para el uso de las redes de transporte y distribución existentes, especialmente en el pago de peajes.

\section{BIBLIOGRAFÍA}

Antúnez, J. F. (1997) - Champalimaud. Temas e Debates, Lisboa.

Cabo Alonso, A. (1991) - Las relaciones interterritoriales: los flujos socioeconómicos y sus repercusiones espaciales. V Coloquio Ibérico de Geografía. Acta, Ponencias y Comunicaciones. Universidad de León, León: 321-331.

CAETANo, L. (1993) - Industrialização dos territórios periféricos: contradições do processo - o caso de Portugal. Cadernos de Geografia, 12, Universidade de Coimbra, Coimbra (13-24).

CASADO, R. (2004) - La larga marcha hacia el mercado ibérico de la electricidad. Diario Expansión, 20 de Enero de 2004, Madrid (4).

Cavaco, C. (1997) - Fronteira Portugal-Espanha e individualidade territorial. Finisterra Revista Portuguesa de Geografia, XXXII (63): 159-166.

Comisión Europea (1999) - Hacia una mayor elección. El mercado único de la electricidad. Oficina de Publicaciones de las Comunidades Europeas. Luxemburgo.

Comisión Nacional de Energía (2003) - Información básica de los sectores de energía. Madrid.

Comisión Nacional de Energía (2003) - Resolución de la Comisión Nacional de Energía sobre la solicitud de autorización presentada por Naturcorp I, S.A. para la fusión de sociedades y posterior constitución de nueva sociedad. Madrid.

Comisión Nacional de Energía (2003) - Resolución de la Comisión Nacional de Energía sobre la solicitud de autorización para la adquisición de una participación del 10 por ciento del capital social de la sociedad portuguesa OMIP por parte de OMEL. Madrid.

Comisión Nacional de Energía; Entidad Reguladora de los Servicios Energéticos (2002) - Modelo de Organización del Mercado Ibérico de Electricidad. Madrid y Lisboa.

De Quinto Romero, J. y Gil Sordo, V. (2002) - El mercado eléctrico ante 2003. Club Español de la Energía y Asociación Española de la Industria Eléctrica, Madrid.

Drain, M. (1994) - L'économie du Portugal. Presses Universitaires de France, Paris.

Eguibide, P. (2004) - Madrid y Lisboa ratifican el nuevo Mercado Ibérico de la Electricidad. Diario El País, 21 de Enero de 2004, Madrid (48).

Endesa (2003) - Informe Anual 2002. Madrid. 
EsPejo MARín, C. (2001) - El sector eléctrico español en la prensa escrita. Cuadernos Geográficos de la Universidad de Granada, 31, Granada: 203-217.

EsPejo MARín, C. (2001) - El proyecto de fusión de Endesa e Iberdrola y su desistimiento. Apuntes sobre el sector eléctrico en España. Nimbus, 7-8, Universidad de Almería, Almería: 51-65.

Espejo Marín, C. (2002) - L'énergie électronucléaire en Espagne. Annales de Géographie, 625, Paris: $319-328$.

Fabra Utray, J. (2004) - ¿Liberalización o regularización? Un mercado para la electricidad. Instituto Pascual Madoz del Territorio, Urbanismo y Medio Ambiente de la Universidad Carlos III de Madrid, Madrid.

Fluxá, F. (1997) - El mercado del sector eléctrico. Revista del Instituto de Estudios Económicos, 1-2, Madrid: 343-365.

García Zarza, E. (1998) - Usos conflictivos del agua entre España y Portugal. Cadernos de Geografia, 17, Universidade de Coimbra, Coimbra: 93-105.

Gaspar, J. (dir.) (1987-1989) - Portugal. Os próximos 20 anos. Ocupação e organização do espaço. Fundação Calouste Gulbenkian, Lisboa.

Gaspar, J. (1993) - As Regiões Portuguesas. Ministério do Planeamento, Lisboa.

GASPAR, J. (1996) - Planeamento transfronteiriço e desenvolvimento regional do sudoeste comunitário. In Campesino Fernández, A. y Velasco Bernardo, C. (coords.) - Portugal-España: Ordenación Territorial del Suroeste Comunitario. Actas, Ponencias y Comunicaciones (VII Coloquio Ibérico de Geografía). Universidad de Extremadura, Salamanca (399-410).

Hidrocantábrico (2003) - Informe Anual 2002. Oviedo.

IBERDRola (2003) - Informe Anual 2002. Bilbao.

IBERDROLA (2004) - Informe Anual 2003. Bilbao.

JACINTO, R. (1995) - As regiões portuguesas de fronteira: perspectivas de desenvolvimento e de cooperação transfronteriza. Cadernos de Geografia, 14, Universidade de Coimbra, Coimbra: $37-54$.

Medeiros, C. A. (1991) - Geografía Humana de Portugal. Oikos-Tau, Barcelona.

Ministerio de Economía (2002) - Planificación de los Sectores de Electricidad y Gas. Desarrollo de las Redes de Transporte 2002-2011. Secretaría de Estado de Energía, Madrid.

Ministerio de Economía (2003) - La energía en España en 2002. Dirección General de Política Energética y Minas, Madrid.

Molina Ibáñez, M. (2001) - Las fuentes de energía. In Gil Olcina, A. y Gómez Mendoza, J. (coords.) - Geografía de España. Ariel, Barcelona: 455-476.

Monforte, C. (2004) - Iberdrola recibe activos de gas en Portugal tras firmar la paz con EDP. Diario Cinco Días, 7 de Febrero de 2004, Madrid (3).

Morales Gil, A. (1996) - Panificación peninsular de los usos del agua. In Campesino Fernández, A. y Velasco Bernardo, C. (coords.) - Portugal-España: Ordenación Territorial del Suroeste Comunitario. Actas, Ponencias y Comunicaciones (VII Coloquio Ibérico de Geografía). Universidad de Extremadura, Salamanca: 261-268. 
Moreira da Silva, R. F. (1996) - Possíveis implicações do Plano Hidrológico Espanhol nos recursos hídricos portugueses (notas de reflexão). In Campesino Fernández, A. y Velasco Bernardo, C. (coords.) - Portugal-España: Ordenación Territorial del Suroeste Comunitario. Actas, Ponencias y Comunicaciones (VII Coloquio Ibérico de Geografía). Universidad de Extremadura, Salamanca: 269-282.

Porto, M. (1996) - O ordenamento do território face aos desafios da competitividade. In Dinamismo sócio-económicos e (re)organização territorial: processos de urbanização e de restructuração productiva. Universidade de Coimbra, Coimbra: 643-706.

Red Eléctrica de España (2003) - El Sistema Eléctrico Español. Informe 2002. Madrid.

Ribeiro, O.; Lautensach, H.; Daveau, S. (1987-1991) - Geografia de Portugal. 4 vols. João Sá da Costa, Lisboa.

Silva, E. e SANTos, A. (1991) - Alguns aspectos geográficos do consumo em Portugal. In V Coloquio Ibérico de Geografía. Acta, Ponencias y Comunicaciones. Universidad de León, León: 543-550.

SoEIRo De BRIto, R. (dir.) (1994) - Portugal. Perfil geográfico. Editorial Estampa, Lisboa.

SUDRIÀ TRIAY, C. (1990) - La industria eléctrica y el desarrollo económico de España. In Electricidad y desarrollo económico: Perspectiva histórica de un siglo. Hidroeléctrica del Cantábrico, S.A.: 75 Aniversario. Hidroeléctrica del Cantábrico, Oviedo: 147-184.

Unesa (2003) - Memoria Estadística. Informe sobre las Actividades Eléctricas. 2002. Madrid.

Unión Fenosa (2003) - Informe Anual 2002. Madrid.

YÁrnoz, C. (2004) - Iberdrola cambia el 4\% de Galp por activos de gas en Portugal. Diario El País, 7 de Febrero de 2004, Madrid (52). 